MATHEMATICS OF COMPUTATION

Volume 76, Number 257, January 2007, Pages 251-272

S 0025-5718(06)01881-3

Article electronically published on August 22, 2006

\title{
A LAX-WENDROFF TYPE THEOREM FOR UNSTRUCTURED QUASI-UNIFORM GRIDS
}

\author{
VOLKER ELLING
}

\begin{abstract}
A well-known theorem of Lax and Wendroff states that if the sequence of approximate solutions to a system of hyperbolic conservation laws generated by a conservative consistent numerical scheme converges boundedly a.e. as the mesh parameter goes to zero, then the limit is a weak solution of the system. Moreover, if the scheme satisfies a discrete entropy inequality as well, the limit is an entropy solution. The original theorem applies to uniform Cartesian grids; this article presents a generalization for quasi-uniform grids (with Lipschitz-boundary cells) uniformly continuous inhomogeneous numerical fluxes and nonlinear inhomogeneous sources. The added generality allows a discussion of novel applications like local time stepping, grids with moving vertices and conservative remapping. A counterexample demonstrates that the theorem is not valid for arbitrary non-quasi-uniform grids.
\end{abstract}

\section{INTRODUCTION}

Consider the Cauchy problem for systems of first-order conservation laws,

$$
\begin{aligned}
\sum_{i=0}^{d} \frac{d}{d y_{i}} f_{i}(u(y), y) & =p(u(y), y) \quad\left(y \in \mathbb{R}_{+}^{d+1}\right), \\
u(0, x) & =u_{0}(x) \quad\left(x \in \mathbb{R}^{d}\right),
\end{aligned}
$$

where $\mathbb{R}_{+}^{d+1}:=(0, \infty) \times \mathbb{R}^{d}, u: \mathbb{R}_{+}^{d+1} \rightarrow P$ (where $P \subset \mathbb{R}^{m}$ is a bounded open subset of the set of physically admissible values $), f=\left(f_{0}, \ldots, f_{d}\right)$ with $f_{0}(w)=w$ and smooth fluxes $f_{i}=\left(f_{i 1}, \ldots, f_{i m}\right): P \times \overline{\mathbb{R}_{+}^{d+1}} \rightarrow \mathbb{R}^{m}(i=1, \ldots, d)$, smooth source $p=\left(p_{1}, \ldots, p_{m}\right): P \times \overline{\mathbb{R}_{+}^{d+1}} \rightarrow \mathbb{R}^{m}$, and initial values $u_{0}: \mathbb{R}^{d} \rightarrow P$.

For the analysis of initial-value problems it is common to separate the time variable and the spatial variable(s); however, for the purposes of the Lax-Wendroff theorem there is no benefit in distinguishing them. For brevity of notation we collect them in the single vector $y=(t, x) \in \mathbb{R}_{+}^{d+1}$. $x$ will be used for coordinates in $\mathbb{R}^{d} ; V$, resp. $S$, refer to the $(d+1)$-dimensional, resp. $d$-dimensional, Hausdorff measure.

Received by the editor April 21, 2003 and, in revised form, October 20, 2005.

2000 Mathematics Subject Classification. Primary 65M12; Secondary 35L65.

Key words and phrases. Finite volume method, conservation law, convergence, Lax-Wendroff, conservative remapping.

This material is based upon work supported by an SAP/Stanford Graduate Fellowship and by the National Science Foundation under Grant no. DMS 0104019. Any opinions, findings, and conclusions or recommendations expressed in this material are those of the author and do not necessarily reflect the views of the National Science Foundation.

(C)2006 Volker Elling 
It is well known that even for smooth initial values $u_{0}$, there need not exist a smooth solution to (1) for all $y_{0}>0$. It is necessary to extend the search to weak solutions, i.e., to $u \in L^{1}\left(\mathbb{R}_{+}^{d+1} ; P\right)$ that satisfy

$$
-\int_{\mathbb{R}_{+}^{d+1}} \sum_{i=0}^{d} f_{i}(u(y), y) \frac{\partial \phi}{\partial y_{i}}(y) d V(y)=\int_{\mathbb{R}^{d}} \phi(0, \cdot) u_{0} d S+\int_{\mathbb{R}_{+}^{d+1}} p(u(y), y) \phi(y) d V(y)
$$

for all $\phi \in C_{c}^{\infty}\left(\overline{\mathbb{R}_{+}^{d+1}}\right)$. Since there can be more than one weak solution, an entropy condition is needed to select the "physical" one: let $\eta=\left(\eta_{0}, \ldots, \eta_{d}\right)^{\prime}, \eta_{i}: P \times$ $\overline{\mathbb{R}_{+}^{d+1}} \rightarrow \mathbb{R}$ smooth, $\eta_{0}$ (the entropy) strictly convex, and $\eta_{1}, \ldots, \eta_{d}$ (entropy fluxes) such that

$$
\frac{\partial \eta_{i}}{\partial u_{\alpha}}=\sum_{\beta=1}^{m} \frac{\partial \eta_{0}}{\partial u_{\beta}} \frac{\partial f_{i \beta}}{\partial u_{\alpha}} \quad(i=1, \ldots, d, \alpha=1, \ldots, m)
$$

$\eta$ is called the entropy/entropy flux pair. The entropy condition is

$$
\sum_{i=0}^{d} \frac{d}{d y_{i}} \eta_{i}(u(y), y) \leq \underbrace{\sum_{\beta=1}^{m} \frac{\partial \eta_{0}}{\partial u_{\beta}}(u(y), y) p_{\beta}(u(y), y)+\sum_{i=0}^{d} \frac{\partial \eta_{i}}{\partial y_{i}}(u(y), y)}_{=: g(u(y), y)}
$$

which is meant to hold in the weak sense, i.e., for all nonnegative $\phi \in C_{c}^{\infty}\left(\overline{\mathbb{R}_{+}^{d+1}}\right)$

$$
\begin{aligned}
-\int_{\mathbb{R}_{+}^{d+1}} \sum_{i=0}^{d} \eta_{i}(u(y), y) \frac{\partial \phi}{\partial y_{i}}(y) d V(y) \leq & \int_{\mathbb{R}^{d}} \eta_{0}\left(u_{0}(\cdot)\right) \phi(0, \cdot) d V \\
& +\int_{\mathbb{R}_{+}^{d+1}} \phi(y) g(u(y), y) d V .
\end{aligned}
$$

(Note: whether this entropy condition is sufficient to guarantee uniqueness is not known, except for some special cases.)

The rest of this section is limited to the case of conservation laws without sources; the presence of sources, as in a reactive flow, poses additional difficulties.

The classical proof that a sequence $\left(u^{h}\right)$ of numerical approximations to (1) converges to an entropy solution proceeds as follows: the properties of the numerical scheme (e.g., a monotone conservative scheme with consistent homogeneous (i.e., $y$ independent) fluxes on a uniform Cartesian grid; see HHL76], CM80 and [CT80) guarantee that $\left(u^{h}\right)$ is bounded in $L^{\infty}$ and $T V$ (the space of functions with bounded variation in the sense of Tonelli-Cesari). This implies that some subsequences converge pointwise almost everywhere (in the presence of uniform $L^{\infty}$ boundedness equivalent to $L_{\text {loc }}^{1}$ convergence); the Lax-Wendroff theorem (see [LW60]) proves that the limits of these subsequences are indeed weak solutions of (1). Moreover, if the $u^{h}$ satisfy discrete entropy inequalities, then the limits must be entropy solutions of (11). Whenever entropy solutions are unique, the entire sequence $\left(u^{h}\right)$ must converge to the entropy solution. Positive uniqueness results are available in special cases (see Kru70 for scalar conservation laws in multiple dimensions or BL97] for 1D system entropy solutions with small total variation and some other restrictions), but see [Ell03] for a possible counterexample for 2D Euler system solutions. 
In many cases, $L_{\text {loc }}^{1}$ precompactness is difficult to prove - and might be false e.g., for unstructured grids or higher-order schemes for scalar conservation laws, not to mention schemes for systems of conservation laws. (For this reason, techniques based on measure-valued solutions which require $L^{\infty}$ boundedness, but not $L_{\text {loc }}^{1}$ precompactness, have been developed and successfully applied to the scalar case in CL91], CL93; see also [Noe95] for irregular grids.) However, CCL94] have generalized earlier work by Kuz75 (see also San83) to a large class of unstructured grids. They prove $L^{1}$ convergence of order $\frac{1}{4}$ to the entropy solution, for monotone numerical fluxes with antidiffusive modifications; the modifications allow for higher order in regions where the entropy solution is smooth. Although these Kuznetsovtype proofs yield convergence without resort to the Lax-Wendroff theorem, they demonstrate that the preconditions of the Lax-Wendroff theorem are satisfied more often than previously thought.

More importantly, while rigorous proofs of convergence are limited to special cases, observing actual output of good numerical schemes suggests that bounded a.e. convergence is rather common, even for important systems like compressible gas dynamics. In this sense, the Lax-Wendroff theorem has important heuristic value: it guarantees that the limit, if there is one, is a weak solution; moreover, in the presence of a discrete entropy condition, it guarantees that the limit is an entropy solution. Finally, the Lax-Wendroff theorem serves as a theoretical motivation for focusing on conservative schemes with consistent fluxes (however, occasionally nonconservative schemes are used in practice).

While the Kuznetsov-type proof in CCL94 applies to a large class of unstructured meshes, it relies strongly on special properties of scalar conservation laws; the same holds for techniques based on weak convergence and measure-valued solutions. It seems that only the Lax-Wendroff theorem provides at least a partial result for systems.

The original Lax-Wendroff theorem requires a 1D uniform Cartesian grid, continuous fluxes, $L_{\text {loc }}^{1}$ precompactness, and $L^{\infty}$ boundedness. LeVeque LeV92, Section 12.4] simplifies the proof, at the cost of requiring locally Lipschitz-continuous numerical fluxes and $T V$ boundedness. KRW96 present a proof for 2D polygonal meshes, locally Lipschitz-continuous numerical fluxes, $L^{\infty}$ boundedness, $L_{\text {loc }}^{1}$ precompactness, and an explicit CFL condition; however, their assumptions (2.3) and (2.4) about the mesh seem restrictive. More general triangular meshes are covered by Proposition 4.4.1 in GR96]; it might be possible to extend their proof technique to polygonal meshes. With straightforward modifications to the statement and proof, all of these results and proofs apply to an arbitrary number of dimensions; however, none of them seem to generalize into other directions easily. This article considers a quasi-uniform mesh with no other geometric restrictions, (uniformly) continuous inhomogeneous numerical fluxes, and nonlinear inhomogeneous source terms.

Only the Cauchy problem is discussed; boundary conditions pose many open research problems, both theoretically and numerically. Even in benign cases where the flux is completely prescribed and independent of the solution near the boundary (as in supersonic inflow), one needs to make additional assumptions about the convergence of the numerical solution near the boundary which are not implied by mere boundedly a.e. convergence. 
Section 2 introduces the grids, numerical fluxes, and numerical sources and the conditions imposed on them; this rather abstract framework is illustrated by a simple example in Section 2.6. Section 3 contains the statement and proof of the generalized Lax-Wendroff theorem (Theorem 1). Section 4 provides a counterexample that explains why Theorem [1] does not always hold for Section 12.4 non-quasi-uniform grids. The newfound generality enables theoretical discussion of some numerical techniques and applications in Section 5 .

\section{Notation AND ASsUmptions}

2.1. Landau symbols. Two sequences of grids will be used: unstructured grids with parameter $h$, and uniform Cartesian grids with parameter $H$. An expression $A$ is said to be $O(B)$ ( $B$ is some other expression) if there is some constant $c$, independent of

$$
C, N, F, h, H, \epsilon, \rho, k, w
$$

so that

$$
A \leq c B
$$

as long as $h, H \in(0,1]$ and as long as

$$
\rho:=\frac{h}{H}<\frac{1}{2}
$$

$A$ is said to be $\Omega(B)$ if $B$ is $O(A)$.

We say that an expression is $o_{\rho, \epsilon}(1)$ if, for any fixed values of $\rho, \epsilon>0$ (and $h:=\rho H)$, it converges to 0 as $H \downarrow 0$.

2.2. Grids. For any $h>0$, let $\mathcal{C}^{h}$ be a system of closed subsets (called cells) of $\mathbb{R}_{+}^{d+1}$ with pairwise disjoint interiors so that

$$
\bigcup_{C \in \mathcal{C}^{h}} C=\overline{\mathbb{R}_{+}^{d+1}} .
$$

We require the cells to have Lipschitz boundaries; this is more than weak enough for all conceivable numerical meshes. For $C, N \in \mathcal{C}^{h}$ with $S(C \cap N)>0$, let $C \rightarrow N$ denote the ordered pair $(C, N)$; depending on the context it will refer to $C \cap N$ instead. The unit normal $n_{C \rightarrow N}(y)$ in each point $y \in C \cap N$ is fixed as pointing into $N$. The $C \rightarrow N$ are called interior faces; the other class of faces consists of initial faces $C \rightarrow \partial, \partial \rightarrow C$ (where $\left.C \cap\left(\{0\} \times \mathbb{R}^{d}\right) \neq \emptyset\right) . \partial \rightarrow C$ will sometimes refer to $C \cap\left(\{0\} \times \mathbb{R}^{d}\right)$, with unit normal $(1,0, \ldots, 0)^{\prime} ; C \rightarrow \partial$ will refer to the same surface with opposite unit normal. Define $\hat{\mathcal{C}}^{h}:=\mathcal{C}^{h} \cup\{\partial\}$. Let $\mathcal{F}^{h}$ be the set of interior faces, $\hat{\mathcal{F}}^{h}$ the set of all faces.

To "define" the mesh parameter $h$, require

$$
\operatorname{diam} C \leq h \quad\left(C \in \mathcal{C}^{h}\right)
$$

this implies $V(C) \leq h^{d+1}$. On the other hand, the mesh must be quasi-uniform in the following sense:

$$
V(C)=\Omega\left(h^{d+1}\right) \quad\left(C \in \mathcal{C}^{h}\right) .
$$

Moreover, the cell surface measure must be controlled:

$$
S(\partial C)=O\left(h^{d}\right) \quad\left(C \in \mathcal{C}^{h}\right) .
$$


Let $\mathcal{B}^{h}$ be the $\sigma$-algebra generated by $\mathcal{C}^{h}$ over $\mathbb{R}_{+}^{d+1}$; the elements of $M\left(\mathcal{B}^{h} ; P\right)$ (the space of $\mathcal{B}^{h}$-Borel-measurable maps on $\mathbb{R}_{+}^{d+1}$ into $P$ ) are called grid functions. For $u^{h} \in M\left(\mathcal{B}^{h} ; P\right)$, let $u_{C}^{h} \in P$ denote the constant value of $u^{h}$ on $\operatorname{int} C\left(C \in \mathcal{C}^{h}\right)$.

2.3. Numerical fluxes. Over every face $F \in \hat{\mathcal{F}}^{h}$ there is a numerical flux $E_{F}$ : $M\left(\mathcal{B}^{h}\right) \rightarrow \mathbb{R}$. (Note: the following definitions make sense for numerical entropy fluxes. The usual numerical fluxes can be reduced to this case; see Section [2.6.) The following requirements are imposed on numerical fluxes over interior faces:

(1) Consistency: for $w \in P$, let $\hat{w}$ be the constant grid function with value $w$ (i.e., $\hat{w}_{C}=w$ for all $C \in \mathcal{C}^{h}$ ). We require

$$
E_{C \rightarrow N}(\hat{w})=\int_{C \rightarrow N} \eta(w, y) \cdot n_{C \rightarrow N}(y) d S(y) .
$$

(2) Uniform continuity: there is a function $\delta_{E}:(0, \infty) \rightarrow(0, \infty)$ so that

$$
\begin{aligned}
& \forall \epsilon>0, h>0, F \in \mathcal{F}^{h}, w \in \mathbb{R}^{m}, w^{h} \in L^{\infty}\left(\mathcal{B}^{h} ; P\right): \\
& \left\|w^{h}-\hat{w}\right\|_{L^{\infty}\left(\mathcal{B}^{h} ; P\right)} \leq \delta_{E}(\epsilon) \Rightarrow\left|E_{F}\left(w^{h}\right)-E_{F}(\hat{w})\right| \leq \epsilon h^{d}
\end{aligned}
$$

(again, $\hat{w}$ denotes the constant grid function with value $w$ ).

(3) Uniform boundedness: for any 11 sequence $\left(w^{h}\right)_{h>0}$,

$$
E_{F}\left(w^{h}\right)=O\left(h^{d}\right) \quad\left(F \in \mathcal{F}^{h}\right) .
$$

(4) Conservativeness: for all $w^{h} \in M\left(\mathcal{B}^{h} ; P\right), C \rightarrow N \in \mathcal{F}^{h}$,

$$
E_{C \rightarrow N}\left(w^{h}\right)=-E_{N \rightarrow C}\left(w^{h}\right) .
$$

(5) Bounded stencil: define the stencil of $F \in \mathcal{F}^{h}$ as

$$
\operatorname{stn} F:=\left\{C \in \mathcal{C}^{h}: w^{h} \mapsto E_{F}\left(w^{h}\right) \text { not constant in } w_{C}^{h}\right\}
$$

and require

$$
\sup _{C \in \operatorname{stn} F} d(C, F)=O(h) .
$$

For initial faces, we impose the numerical initial condition

$$
E_{\partial \rightarrow C}\left(w^{h}\right)=-E_{C \rightarrow \partial}\left(w^{h}\right)=\int_{\partial \rightarrow C} \eta_{0}\left(u_{0}(x), 0, x\right) d S(x) \quad \forall w^{h} \in M\left(\mathcal{B}^{h} ; \mathbb{R}^{m}\right) .
$$

2.4. Numerical sources. The source terms in (6) are approximated by numerical sources: functions $G_{C}: M\left(\mathcal{B}^{h} ; P\right) \rightarrow \mathbb{R}$ for each cell $C \in \mathcal{C}^{h}$. The numerical sources must satisfy the following conditions (that are very similar to the ones for numerical fluxes):

(1) Consistency:

$$
\forall w \in P, C \in \mathcal{C}^{h}: G_{C}(\hat{w})=\int_{C} g(w, y) d V(y)
$$

(where $\hat{w}$ is the constant grid function with value $w \in \mathbb{R}^{m}$ ).

\footnotetext{
${ }^{1}$ To apply this to common cases, restrict $P$ to be bounded (scalar case), bounded away from vacuum (gas dynamics), etc.
} 
(2) Uniform continuity: there is a function $\delta_{G}:(0, \infty) \rightarrow(0, \infty)$ so that

$$
\begin{aligned}
& \forall \epsilon>0, h>0, C \in \mathcal{C}^{h}, w \in P, w^{h} \in L^{\infty}\left(\mathcal{B}^{h} ; P\right): \\
& \left\|w^{h}-\hat{w}\right\|_{L^{\infty}\left(\mathcal{B}^{h} ; P\right)} \leq \delta_{G}(\epsilon) \Rightarrow\left|G_{C}\left(w^{h}\right)-G_{C}(\hat{w})\right| \leq \epsilon h^{d+1} .
\end{aligned}
$$

(3) Uniform boundedness: for any sequence $\left(w^{h}\right)_{h>0}$,

$$
G_{C}\left(w^{h}\right)=O\left(h^{d+1}\right) \quad\left(C \in \mathcal{C}^{h}\right) .
$$

(4) Bounded stencil: define the stencil of $C \in \mathcal{C}^{h}$ as

$$
\operatorname{stn} C:=\left\{C^{\prime} \in \mathcal{C}^{h}: w^{h} \mapsto G_{C}\left(w^{h}\right) \text { not constant in } w_{C^{\prime}}^{h}\right\}
$$

and require

$$
\sup _{C^{\prime} \in \operatorname{stn} C} d\left(C, C^{\prime}\right)=O(h)
$$

\subsection{Result.}

Theorem 1. If a sequence $\left(u^{h}\right)_{h>0}$ of grid functions satisfies the discrete scalar inequalities

$$
\sum_{N \in \hat{\mathcal{C}}^{h}, C \cap N \neq \emptyset} E_{C \rightarrow N}\left(u^{h}\right) \leq G_{C}\left(u^{h}\right) \quad\left(\forall h>0, C \in \mathcal{C}^{h}\right)
$$

and converges almost everywhere to $u$, then $u$ satisfies (6).

2.6. An example. For illustration, the Lax-Friedrichs scheme (for a system $u_{t}+$ $f(u)_{x}=0$ with initial condition $u(0, x)=u_{0}(x)$ on a uniform 1D grid with cell size $h$ and uniform time steps $\lambda h(0<\lambda \leq 1$ constant $))$ is fit into the abstract framework in the previous sections. For $h>0$, let $\mathcal{C}^{h}$ contain the cells $C_{j}^{n}$,

$$
C_{j}^{n}:=[j h,(j+1) h] \times[n \lambda h,(n+1) \lambda h] \quad\left(j \in \mathbb{Z}, n \in \mathbb{N}_{0}\right) .
$$

Numerical fluxes: for $j \in \mathbb{Z}, n \in \mathbb{N}_{0}$,

$$
\begin{aligned}
F_{\partial \rightarrow C_{j}^{0}}\left(w^{h}\right) & :=\int_{0}^{h} u_{0}(j h+y) d y \\
F_{C_{j}^{n} \rightarrow C_{j}^{n+1}}\left(w^{h}\right) & :=h w_{C_{j}^{n+1}}^{h}, \\
F_{C_{j}^{n} \rightarrow C_{j+1}^{n}}\left(w^{h}\right) & :=h\left(\lambda \frac{f\left(w_{C_{j}^{n}}^{h}\right)+f\left(w_{C_{j+1}^{n}}^{h}\right)}{2}-\frac{w_{C_{j+1}^{n}}^{h}-w_{C_{j}^{n}}^{h}}{2}\right) .
\end{aligned}
$$

Numerical sources: all $=0$. It is easy to check that the numerical fluxes satisfy all conditions, in particular consistency. The numerical solutions $u^{h} \in M\left(\mathcal{B}^{d} ; \mathbb{R}^{m}\right)$ are defined by

$$
\begin{aligned}
C_{0}^{j} & :=h^{-1} \int_{j h}^{(j+1) h} u_{0}(x) d S(x), \\
\sum_{N \in \hat{\mathcal{C}}^{h}} F_{N \rightarrow C}\left(u^{h}\right) & =0 \quad\left(\forall h>0, \forall C \in \mathcal{C}^{h}\right),
\end{aligned}
$$

which is exactly the literature definition, using different notation.

(27) is a system of $\mathbb{R}^{m}$-valued equations for $u^{h}$, but it can obviously be converted into $2 \cdot m$ systems of scalar inequalities of the type (21); Theorem 1 applied to each of them separately implies (3), i.e., that $u$ is a weak solution. 
In a similar fashion, it can be verified that the limit is an entropy solution. For Burgers equation $\left(f(u)=\frac{1}{2} u^{2}\right)$, it is sufficient to prove the entropy inequality for the Kružkov family of entropies and entropy fluxes,

$$
\eta_{0}(u)=|u-a|, \quad \eta_{1}(u)=\operatorname{sgn}(u-a) f(u),
$$

where $a \in \mathbb{R}$ is the family parameter. The numerical entropy fluxes

$$
\begin{aligned}
E_{\partial \rightarrow C_{j}^{0}}\left(u^{h}\right) & :=\int_{0}^{h} \eta_{0}\left(u_{0}(j h+y)\right) d y \\
E_{C_{j}^{n} \rightarrow C_{j}^{n+1}}\left(u^{h}\right) & :=h \eta_{0}\left(u_{C_{j}^{n}}^{h}\right), \\
E_{C_{j}^{n} \rightarrow C_{j+1}^{n}}\left(u^{h}\right) & :=\lambda h\left(F_{C_{j}^{n} \rightarrow C_{j+1}^{n}}\left(u^{h} \vee c\right)-F_{C_{j}^{n} \rightarrow C_{j+1}^{n}}\left(u^{h} \wedge c\right)\right)
\end{aligned}
$$

(see [CM80]) are consistent with $\eta$ and satisfy the discrete entropy inequality (21), so Theorem 1 asserts that $u$ is an entropy solution (in the sense (6] ) ).

\section{Proof of Theorem 1}

The proof is based on two essential ideas: first, the original proof in LW60. uses summation by parts (in analogy to the integration by parts used to derive the concept of weak solution); this requires a Cartesian grid. This obstacle is bypassed by approximating cubes with sidelength $H$ in a uniform Cartesian grid by cells in an unstructured grid with parameter $h$; see Figure1. Summation by parts is carried out for these cubes.

Second, since $\left(u^{h}\right)$ converges in $L_{\text {loc }}^{1}\left(\mathbb{R}_{+}^{d+1}\right), u$ and $u^{h}$ will be "close" and "nearly constant" in a suitable neighbourhood of "almost all" cubes (for $h \downarrow 0$ ), so the continuity and consistency properties of numerical fluxes and sources can be exploited. For the "few" remaining "bad" cubes, one can use uniform boundedness of numerical fluxes and sources. The proof will be completed by first fixing a sufficiently small ratio $\rho$ to minimize geometric errors and then choosing a sufficiently small $H>0$ to control integral errors.

3.1. Cubes. Let $e^{(i)} \in \mathbb{Z}^{d+1}$ (with $i \in\{0, \ldots, d\}$ ) be the standard basis vectors, with the $i$ th component $=1$, and all other components $=0$. Omitting the parameter $H$ for readability, define the closed cubes

$$
I_{k}:=H \cdot \prod_{i=0}^{d}\left[k_{i}, k_{i}+1\right] \quad\left(k \in \mathbb{N}_{0} \times \mathbb{Z}^{d}\right)
$$

with faces

$$
\begin{aligned}
& \partial I_{k}^{i+}:=H \cdot\left(\prod_{j=0}^{i-1}\left[k_{j}, k_{j}+1\right] \times\left\{k_{i}+1\right\} \times \prod_{j=i+1}^{d}\left[k_{j}, k_{j}+1\right]\right), \\
& \partial I_{k}^{i-}:=H \cdot\left(\prod_{j=0}^{i-1}\left[k_{j}, k_{j}+1\right] \times\left\{k_{i}\right\} \times \prod_{j=i+1}^{d}\left[k_{j}, k_{j}+1\right]\right) ;
\end{aligned}
$$

note that the interiors of the $I_{k}$ are pairwise disjoint and that

$$
\overline{\mathbb{R}_{+}^{d+1}}=\bigcup_{k \in \mathbb{N}_{0} \times \mathbb{Z}^{d}} I_{k}
$$


moreover

$$
\partial I_{k}=\bigcup_{i=0}^{d}\left(\partial I_{k}^{i-} \cup \partial I_{k}^{i+}\right) \quad\left(k \in \mathbb{N}_{0} \times \mathbb{Z}^{d}\right) .
$$

3.2. Cube approximation. For given $H, h>0(h<H / 2)$ and $k \in \mathbb{N}_{0} \times \mathbb{Z}^{d}$, we select an approximation $\tilde{I}_{k} \subset \mathcal{C}^{h}$ to $I_{k}$ by requiring that

$$
C \cap I_{k} \neq \emptyset \quad \forall C \in \tilde{I}_{k}
$$

and that the sets $\tilde{I}_{k}$ form a partition of $\mathcal{C}^{h}$. (These conditions need not determine $\tilde{I}_{k}$ uniquely; the particular choice is not important. (34) admits the existence of such $\tilde{I}_{k}$ because the $I_{k}$ cover $\overline{\mathbb{R}_{+}^{d+1}}$.)

We also define (for $i \in\{0, \ldots, d\}, k \in \mathbb{N}_{0} \times \mathbb{Z}^{d}$ )

$$
\begin{aligned}
\partial \tilde{I}_{k} & :=\left\{C \rightarrow \partial \in \hat{\mathcal{F}}^{h}: C \in \tilde{I}_{k}\right\} \cup\left\{C \rightarrow N \in \mathcal{F}^{h}: C \in \tilde{I}_{k}, N \notin \tilde{I}_{k}\right\}, \\
\partial \tilde{I}_{k}^{i \pm}: & = \begin{cases}\left\{C \rightarrow N \in \mathcal{F}^{h}: C \in \tilde{I}_{k}, N \in \tilde{I}_{k \pm e^{(i)}}\right\}, & k \pm e^{(i)} \in \mathbb{N}_{0} \times \mathbb{Z}^{d}, \\
\left\{C \rightarrow \partial \in \hat{\mathcal{F}}^{h}: C \in \tilde{I}_{\left(0, k_{1}, \ldots, k_{d}\right)}\right\}, & \text { else. }\end{cases}
\end{aligned}
$$

Note that

however

$$
C \rightarrow N \in \partial \tilde{I}_{k+e^{(i)}}^{i-} \quad \Leftrightarrow \quad N \rightarrow C \in \partial \tilde{I}_{k}^{i+}
$$

$$
\bigcup_{i=0}^{d}\left(\partial \tilde{I}_{k}^{i-} \cup \partial \tilde{I}_{k}^{i+}\right)=\partial \tilde{I}_{k}
$$

is not true in general (see Lemma 3) because some faces belong to "corners" rather than to sides of the approximated cubes (see Figure 1).

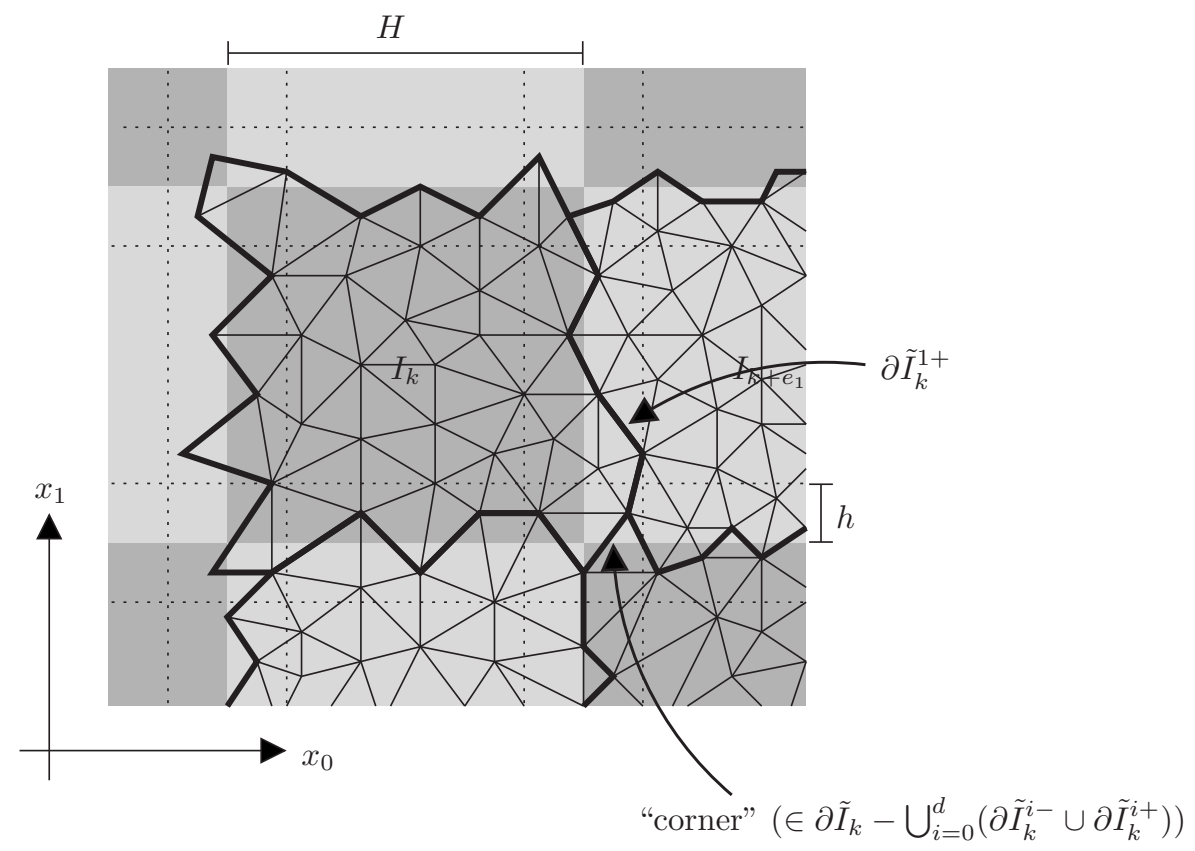

FIGURE 1. A cube $I_{k}$ (checkerboard-shaded grid) is approximated by a cluster (thick boundary) of grid cells (thin triangles). 
3.3. Geometric estimates. For $r>0$ and $A \subset \overline{\mathbb{R}_{+}^{d+1}}$, define the closed neighbourhoods

$$
\bar{Q}_{r}(A):=\left\{y \in \overline{\mathbb{R}_{+}^{d+1}}: d(y, A) \leq r\right\}
$$

Lemma 1.

$$
\bigcup_{C \in \tilde{I}_{k}} C \subset \bar{Q}_{h}\left(I_{k}\right), \quad I_{k} \subset \bar{Q}_{h}\left(\bigcup_{C \in \tilde{I}_{k}} C\right)
$$

SO

$$
V\left(\bigcup_{C \in \tilde{I}_{k}} C\right)=H^{d+1}+O\left(\rho H^{d+1}\right)
$$

Moreover

$$
\begin{aligned}
& \bigcup_{F \in \partial \tilde{I}_{k}^{i \pm}} F \subset \bar{Q}_{h}\left(\partial I_{k}^{i \pm}\right) \\
& \partial I_{k} \subset \bar{Q}_{h}\left(\bigcup_{F \in \partial \tilde{I}_{k}} F\right), \\
& \partial I_{k}^{i \pm} \subset \bar{Q}_{h}\left(\bigcup_{F \in \partial \tilde{I}_{k}^{i \pm}} F\right) .
\end{aligned}
$$

Proof. These are immediate consequences of (8) and of (34), (35), resp. (36).

Lemma 2. $A \subset \mathbb{R}_{+}^{d+1}$ meets $\leq \frac{V\left(\bar{Q}_{h}(A)\right)}{h^{d+1}}$ cells in $\mathcal{C}^{h}$.

Proof. $\operatorname{diam} C \leq h$, so $C \cap A \neq \emptyset$ implies $C \subset \bar{Q}_{h}(A)$. However, (9) implies that $\bar{Q}_{h}(A)$ cannot contain more than $V\left(\bar{Q}_{h}(A)\right) h^{-(d+1)}$ cells.

\section{Corollary 1.}

$$
\begin{array}{r}
\sup _{F \in \hat{\mathcal{F}}^{h}} \# \operatorname{stn} F=O(1), \\
\sup _{C \in \mathcal{C}^{h}} \#\left\{N \in \mathcal{C}^{h}: C \cap N \neq \emptyset\right\}=O(1) ;
\end{array}
$$

for all $k \in \mathbb{N}_{0} \times \mathbb{Z}^{d}$ and $i \in\{0, \ldots, d\}$,

$$
\begin{aligned}
\sup _{k \in \mathbb{N}_{0} \times \mathbb{Z}^{d}} \# \tilde{I}_{k} & =O\left(\rho^{-d-1}\right), \\
\sup _{k \in \mathbb{N}_{0} \times \mathbb{Z}^{d}, i \in\{0, \ldots, d\}, s \in\{+,-\}} \# \partial \tilde{I}_{k}^{i s} & =O\left(\rho^{-d}\right) .
\end{aligned}
$$

Proof. (42): by (15), there is a constant $c$ (independent of $h$ ) so that

$$
\bigcup_{C \in \operatorname{stn} F} C \subset \bar{Q}_{c h}(F)
$$

for all faces $F$. Since $\operatorname{diam} F \leq h, \bar{Q}_{c h}(F)$ is contained in some closed ball with diameter $O(h)$, hence volume $O\left(h^{d+1}\right)$. At most $O(1)$ cells fit into this ball, so $\# \operatorname{stn} F=O(1)$.

(43): this is immediate from Lemma 2

(44): (37) implies

$$
\bar{Q}_{h}\left(\bigcup_{C \in \tilde{I}_{k}} C\right) \subset \bar{Q}_{2 h}\left(I_{k}\right)
$$


hence $\left(\rho \leq \frac{1}{2}\right)$

$$
V\left(\bar{Q}_{h}\left(\bigcup_{C \in \tilde{I}_{k}} C\right)\right)=O\left(H^{d+1}\right) .
$$

Hence by Lemma 2 at most $O\left(\frac{H^{d+1}}{h^{d+1}}\right)=O\left(\rho^{-d-1}\right)$ cells meet $\bigcup_{C \in \tilde{I}_{k}} C$.

(45): from (39) derive

$$
\bar{Q}_{h}\left(\bigcup_{F \in \partial \tilde{I}_{k}^{i \pm}} F\right) \subset \bar{Q}_{2 h}\left(\partial I_{k}^{i \pm}\right)
$$

so

$$
V\left(\bar{Q}_{h}\left(\bigcup_{F \in \partial \tilde{I}_{k}^{i \pm}} F\right)\right)=O\left(h H^{d}\right)=O\left(\rho H^{d+1}\right) .
$$

Hence Lemma 2 shows that at most $O\left(\frac{\rho H^{d+1}}{h^{d+1}}\right)=O\left(\rho^{-d}\right)$ cells meet $\bigcup_{F \in \partial \tilde{I}_{k}^{i \pm}} F$; by (43) each has $O(1)$ faces.

The following lemma states that, for small $\rho$, "most" of $\partial \tilde{I}_{k}$ is composed of the $\partial \tilde{I}_{k}^{i \pm}(i=0, \ldots, d)$, i.e., we can ignore the "corners" of $I_{k}$.

Lemma 3. Define the "half-cylinders"

$$
Z_{k}^{i \pm}:=\left\{y \in \overline{\mathbb{R}_{+}^{d+1}}: y_{i} \gtrless H\left(k_{i}+\frac{1}{2}\right), y_{j} \in H \cdot\left(k_{j}+\rho, k_{j}+1-\rho\right) \quad(j \neq i)\right\}
$$

(see Figure 2). Then

$$
\sum_{F \in \partial \tilde{I}_{k}^{i \pm}} S(F)=\sum_{F \in \partial \tilde{I}_{k}^{i \pm}} S\left(F \cap Z_{k}^{i \pm}\right)+O(\rho) H^{d} .
$$

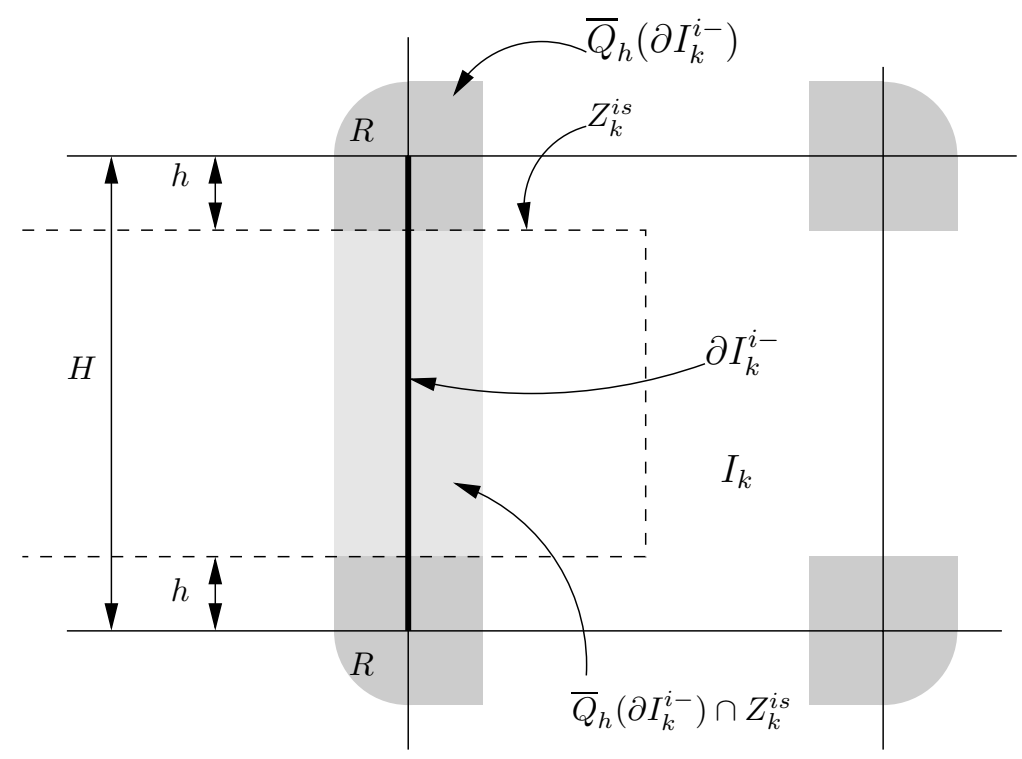

FiguRe 2. $V(R)=O\left(h^{2} H^{d-1}\right)=O\left(\rho^{2} H^{d+1}\right)$. 
Moreover,

$$
\sum_{F \in \partial \tilde{I}_{k}-\bigcup_{i=0}^{d}\left(\partial \tilde{I}_{k}^{i-} \cup \partial \tilde{I}_{k}^{i+}\right)} S(F)=O(\rho) H^{d} .
$$

Proof. (See Figure 2,) Let $F=C \rightarrow N \in \partial \tilde{I}_{k}^{i s}$ for some $i \in\{0, \ldots, d\}, s \in\{+,-\}$ (or $F=C \rightarrow \partial$ ). By (39), whenever $F \cap Z_{k}^{i s} \neq F$, then $F$ (and hence $C$ ) meets

$$
R:=\bar{Q}_{h}\left(\partial I_{k}\right)-\bigcup_{i=0}^{d}\left(Z_{k}^{i+} \cup Z_{k}^{i-}\right) .
$$

However, it is easy to verify that

$$
V\left(\bar{Q}_{h}(R)\right)=O\left(h^{2} H^{d-1}\right)=O\left(\rho^{2} H^{d+1}\right) .
$$

By Lemma 2, at most

$$
O\left(\frac{V\left(\bar{Q}_{h}(R)\right)}{h^{d+1}}\right)=O\left(\rho^{1-d}\right)
$$

cells can meet $R$. By (10), their total surface measure is

$$
=O\left(\rho^{1-d}\right) O\left(h^{d}\right)=O\left(\rho H^{d}\right) ;
$$

this implies (47).

Regarding (48): whenever $C \rightarrow N \in \partial \tilde{I}_{k}$ (with $C \in \tilde{I}_{k}$ ) is not contained in any $\partial \tilde{I}_{k}^{i \pm}$, then $C \rightarrow N$ belongs to a "corner", i.e., $N \in \tilde{I}_{k+m}$ for some $m \in \mathbb{Z}^{d+1}$ with $|m|_{\infty}=1,|m|_{1} \geq 2$ (because of $\rho<\frac{1}{2}$ and $\operatorname{diam} C$, $\operatorname{diam} N \leq h$ ). This means $C \rightarrow N \subset R$; (47) states that these faces may be ignored at a cost of surface measure of $O\left(\rho H^{d}\right)$.

$C \rightarrow \partial \in \partial \tilde{I}_{k}^{0-}$ for some $k \in\{0\} \times \mathbb{Z}^{d}$, so initial faces do not contribute to the sum in (48).

The numerical fluxes over $\bigcup_{F \in \partial \tilde{I}_{k}^{i \pm}} F$ will be pieced together to approximate the exact flux over $\partial I_{k}^{i \pm}$. This requires the following geometric estimate.

Lemma 4. For all $k \in \mathbb{N}_{0} \times \mathbb{Z}^{d}, i \in\{0, \ldots, d\}$,

$$
\left|\left(\int_{\bigcup_{F \in \partial \tilde{I}_{k}^{i-}} F}-\int_{\partial I_{k}^{i-}}\right) n d S\right|=O(\rho) H^{d} .
$$

Proof. (See Figure 3,)

$$
\begin{aligned}
& \left(\int_{\bigcup_{F \in \partial \tilde{I}_{k}^{i-}} F}-\int_{\partial I_{k}^{i-}}\right) n d S \\
& \stackrel{(48)}{=}\left(\int_{\bigcup_{F \in \partial \tilde{I}_{k}^{i-}} F \cap Z_{k}^{i-}}-\int_{\partial I_{k}^{i-} \cap Z_{k}^{i-}}\right) n d S+O(\rho) H^{d} .
\end{aligned}
$$

The first summand in (51) equals

$$
\left(\int_{\partial\left(\cup_{C \in \tilde{I}_{k} C} \cap Z_{k}^{i-}\right)}-\int_{\partial\left(I_{k} \cap Z_{k}^{i-}\right)}\right) n d S=0
$$




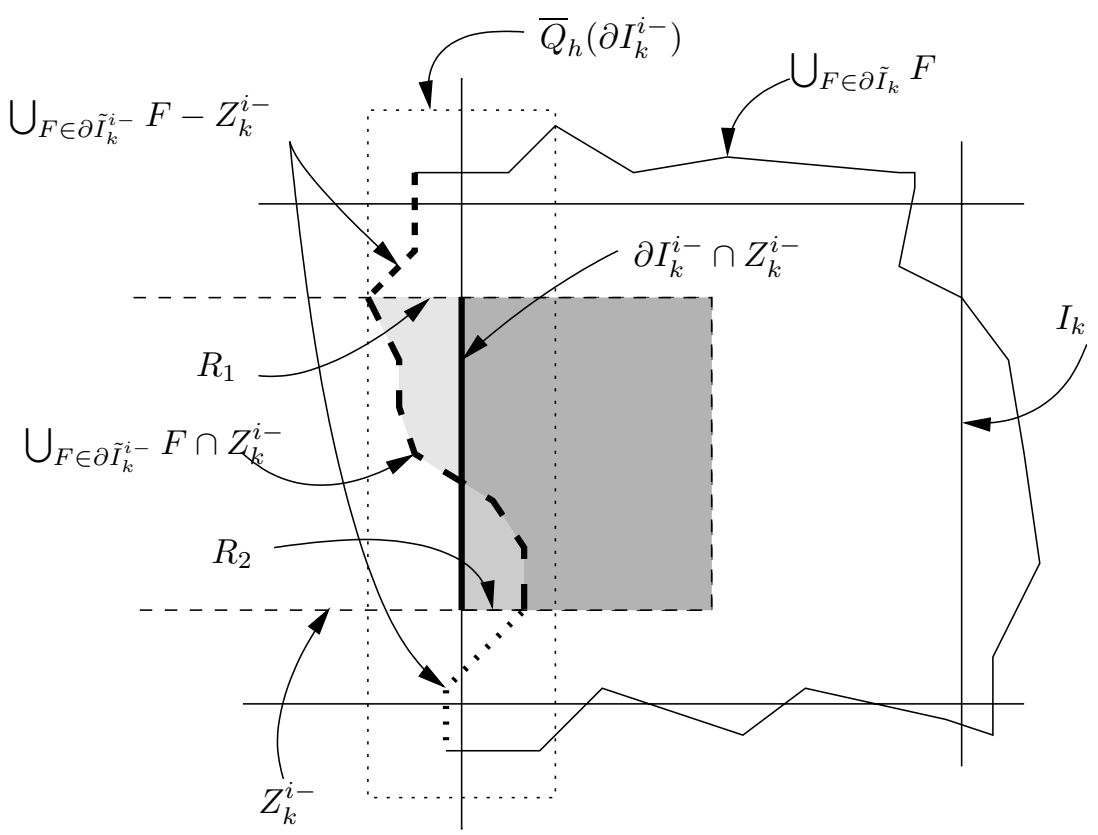

Figure 3 . The surfaces $R_{1}, R_{2}, \bigcup_{F \in \partial \tilde{I}_{k}^{i-}} F-Z_{k}^{i-}$, and $\partial I_{k}^{i-}-Z_{k}^{i-}$ have measure $O(\rho) H^{d}$ and can be neglected.

up to

$$
\int_{R_{1}} n d S-\int_{R_{2}} n d S
$$

where $R_{1}, R_{2}$ are contained in

$$
\partial Z_{k}^{i-} \cap \bar{Q}_{h}\left(\partial I_{k}^{i-}\right),
$$

a set with total surface measure $O(\rho) H^{d}$; hence the two integrals in (52) are $O(\rho) H^{d}$, too.

3.4. Completion of the proof. Consider an arbitrary nonnegative test function $\phi \in C_{c}^{\infty}\left(\overline{\mathbb{R}_{+}^{d+1}}\right)$. Since $\phi$ has compact support, it is sufficient to consider the finite subsets

$$
K:=\left\{k \in \mathbb{N}_{0} \times \mathbb{Z}^{d}: \exists \ell \in \mathbb{Z}^{d+1}:|\ell| \leq 1, \operatorname{supp} \phi \cap I_{k+\ell} \neq \emptyset\right\}
$$

of cube indices. Note that

$$
\# K=O\left(\rho^{-(d+1)}\right)
$$

Define

$$
\begin{aligned}
\operatorname{stn} k & :=\bigcup_{F \in \partial \tilde{I}_{k}} \operatorname{stn} F \cup \bigcup_{C \in \tilde{I}_{k}} \operatorname{stn} C, \\
\operatorname{stn} K & :=\bigcup_{k \in K} \operatorname{stn} k .
\end{aligned}
$$


Lemma 5. For all $w \in L_{\mathrm{loc}}^{1}\left(\overline{\mathbb{R}_{+}^{d+1}}\right)$,

$$
\sum_{k \in K} \int_{\bigcup_{C \in \operatorname{stn} k} C}|w(y)| d V(y)=O(1) \int_{\bigcup_{C \in \operatorname{stn} K} C}|w(y)| d V(y) .
$$

Proof. Due to bounded stencils (15), resp. (20), bounded diameters (8) and $\rho \leq \frac{1}{2}$ (see (7)),

$$
\sup _{C \in \mathcal{C}^{h}} \#\{k \in K: C \in \operatorname{stn} k\}=O(1) .
$$

Hence

$$
\begin{aligned}
\sum_{k \in K} \int_{\cup_{C \in \operatorname{stn} k} C}|w(y)| d V(y) & =\sum_{C \in \operatorname{stn} K} \#\{k \in K: C \in \operatorname{stn} k\} \int_{C}|w(y)| d V(y) \\
& \leq O(1) \sum_{C \in \operatorname{stn} K} \int_{C}|w(y)| d V(y) .
\end{aligned}
$$

We need to show that $u, u^{h}$ are "almost constant" and "close" on "most" cubes. We introduce a new parameter $\epsilon>0$. Again omitting $\epsilon, \rho, H>0$ from the symbols for readability, define

$$
\begin{aligned}
\bar{u}_{k} & :=V\left(I_{k}\right)^{-1} \int_{I_{k}} u(y) d V(y), \\
B_{1} & :=\left\{k \in K: \exists C \in \operatorname{stn} k:\left|u_{C}^{h}-\bar{u}_{k}\right|>\min \left\{\delta_{E}(\epsilon), \delta_{G}(\epsilon)\right\}\right\}, \\
B_{2} & :=\left\{k \in K: \frac{V\left\{y \in I_{k}:\left|u(y)-\bar{u}_{k}\right|>\min \left\{\delta_{E}(\epsilon), \delta_{G}(\epsilon)\right\}\right\}}{V\left(I_{k}\right)}>\epsilon\right\}, \\
B & :=B_{1} \cup B_{2}, \\
G & :=K-B ;
\end{aligned}
$$

$B$ contains the "bad", and $G$ the "good" cube indices.

Lemma 6. For any choice of $\epsilon, \rho>0$

$$
\frac{\# B}{H^{-(d+1)}}=o_{\rho, \epsilon}(1)
$$

Proof. $B_{1}$ and $B_{2}$ are treated separately. First $B_{2}$ : let $w \in C^{\infty}\left(\overline{\mathbb{R}_{+}^{d+1}}\right)$ be arbitrary (it will approximate $u$ ); define

$$
\bar{w}_{k}=V\left(I_{k}\right)^{-1} \int_{I_{k}} w(y) d V(y) .
$$

Then

$$
\begin{aligned}
\sum_{k \in B_{2}} & \int_{I_{k}}\left|u(y)-\bar{u}_{k}\right| d V(y) \leq \sum_{k \in K} \int_{I_{k}}\left|u(y)-\bar{u}_{k}\right| d V(y) \\
\leq & \sum_{k \in K} \int_{I_{k}}|u(y)-w(y)| d V(y) \\
& +\sum_{k \in K} \int_{I_{k}}\left|w(y)-\bar{w}_{k}\right| d V(y)+\sum_{k \in K} V\left(I_{k}\right)\left|\bar{w}_{k}-\bar{u}_{k}\right| \\
= & O\left(\|u-w\|_{L^{1}\left(\cup_{k \in K} I_{k}\right)}+\|D w\|_{L^{\infty}\left(\cup_{k \in K} I_{k}\right)} H\right)
\end{aligned}
$$


because

$$
\begin{aligned}
& \sum_{k \in K} V\left(I_{k}\right)\left|\bar{u}_{k}-\bar{w}_{k}\right|=\sum_{k \in K}\left|\int_{I_{k}} u(y)-w(y) d V(y)\right| \\
& \leq \sum_{k \in K} \int_{I_{k}}|u(y)-w(y)| d V(y) \leq\|u-w\|_{L^{1}\left(\bigcup_{k \in K} I_{k}\right)}
\end{aligned}
$$

and because

$$
\left|\bar{w}_{k}-w(y)\right|=O\left(H\|D w\|_{L^{\infty}\left(\bigcup_{k \in K} I_{k}\right)}\right.
$$

for $y \in I_{k}$, by smoothness of $w$. For each $k \in B_{2}$,

$$
\int_{I_{k}}\left|u(y)-\bar{u}_{k}\right| d V(y) \geq \epsilon \min \left\{\delta_{E}(\epsilon), \delta_{G}(\epsilon)\right\} V\left(I_{k}\right),
$$

by definition (58) of $B_{2}$, so (61) implies that

$$
\# B_{2} \cdot H^{d+1}=\sum_{k \in B_{2}} V\left(I_{k}\right)=O\left(\frac{\|u-w\|_{L^{1}\left(\bigcup_{k \in K} I_{k}\right)}+\|D w\|_{L^{\infty}\left(\bigcup_{k \in K} I_{k}\right)} H}{\epsilon \min \left\{\delta_{E}(\epsilon), \delta_{G}(\epsilon)\right\}}\right) .
$$

By first choosing $w \in C^{\infty}\left(\mathbb{R}_{+}^{d+1}\right)$ with sufficiently small $\|u-w\|_{L^{1}\left(\bigcup_{k \in K} I_{k}\right)}$ and then choosing an upper bound for $H$, the right-hand side can be made arbitrarily small.

Regarding $B_{1}$,

$$
\begin{aligned}
& \sum_{k \in B_{1}} \int_{\cup_{C \in \operatorname{stn} k} C}\left|u^{h}(y)-\bar{u}_{k}\right| d V(y) \\
& \leq \sum_{k \in K} \int_{\bigcup_{C \in \operatorname{stn} k} C}\left|u^{h}(y)-\bar{u}_{k}\right| d V(y) \\
& \leq \sum_{k \in K} \int_{\bigcup_{C \in \operatorname{stn} k} C}\left|u^{h}(y)-u(y)\right| d V(y) \\
& \quad+\sum_{k \in K} \int_{\bigcup_{C \in \operatorname{stn} k} C}|u(y)-w(y)| d V(y) \\
& \quad+\sum_{k \in K} \int_{\bigcup_{C \in \operatorname{stn} k} C}\left|w(y)-\bar{w}_{k}\right| d V(y) \\
& \quad+\sum_{k \in K} \int_{\bigcup_{C \in \operatorname{stn} k} C}\left|\bar{w}_{k}-\bar{u}_{k}\right| d V(y) \\
& \stackrel{(55)}{=} O\left(\left\|u^{h}-u\right\|_{L^{1}}\left(\cup_{C \in \operatorname{stn} K} C\right)\right. \\
& \left.\quad+\|u-w\|_{L^{1}\left(\cup_{C \in \operatorname{stn} K} C\right)}+H\|D w\|_{L^{\infty}\left(\cup_{C \in \operatorname{stn} K} C\right)}\right) .
\end{aligned}
$$

For each $k \in B_{1}$,

$$
\begin{aligned}
\int_{\bigcup_{C \in \operatorname{stn} k} C}\left|u^{h}(y)-\bar{u}_{k}\right| d V(y) & =\Omega\left(\rho^{d+1} H^{d+1} \min \left\{\delta_{E}(\epsilon), \delta_{G}(\epsilon)\right\}\right) \\
& =\Omega\left(\rho^{d+1} V\left(I_{k}\right) \min \left\{\delta_{E}(\epsilon), \delta_{G}(\epsilon)\right\}\right),
\end{aligned}
$$


so (64) (with (91) ) shows

$$
\begin{aligned}
& \# B_{1} \cdot H^{d+1} \\
& =O\left(\frac{\left\|u^{h}-u\right\|_{L^{1}\left(\bigcup_{C \in \operatorname{stn} K} C\right)}+\|u-w\|_{L^{1}\left(\bigcup_{C \in \operatorname{stn} K} C\right)}+\|D w\|_{L^{\infty}\left(\bigcup_{C \in \operatorname{stn} K} C\right)} H}{\rho^{d+1} \min \left\{\delta_{E}(\epsilon), \delta_{G}(\epsilon)\right\}}\right) .
\end{aligned}
$$

By first choosing a suitable $w$ and then choosing an upper bound for $H$, the righthand side can be made arbitrarily small.

Now we can finish the proof of Theorem 1. Sum (21) over $C \in \tilde{I}_{k}$, multiply with $\phi(H k)(\geq 0)$, and sum over $k \in K$ :

$$
\sum_{k \in K} \phi(H k) \sum_{C \in \tilde{I}_{k}} G_{C}\left(u^{h}\right) \geq \sum_{k \in K} \phi(H k) \sum_{F \in \partial \tilde{I}_{k}} E_{F}\left(u^{h}\right)
$$

(here we used the conservation property (14) to eliminate $F \notin \bigcup_{k \in K} \partial \tilde{I}_{k}$, i.e., $F=C \rightarrow N$ with $C, N \in \tilde{I}_{k}$ for the same $k$ ).

Collecting the terms on the right-hand side of (65) where $F$ is an initial face yields

$$
\sum_{k \in K} \sum_{C \rightarrow \partial \in \partial \tilde{I}_{k}^{0-}} E_{C \rightarrow \partial}\left(u^{h}\right) \phi(H k)
$$

which equals

$$
-\int_{\mathbb{R}^{d}} \eta_{0}\left(u_{0}(x)\right) \phi(0, x) d S(x)+O(H),
$$

using (16) and smoothness plus compact support of $\phi$.

There are at most two terms per interior face in (65); they can be written (using (14)) as

$$
E_{C \rightarrow N}\left(u^{h}\right)\left(\phi\left(H k_{C}\right)-\phi\left(H k_{N}\right)\right)
$$

where $C \in \tilde{I}_{k_{C}}, N \in \tilde{I}_{k_{N}}\left(\left|k_{C}-k_{N}\right|_{\infty}=1\right)$.

Note that the $\phi$ difference is $O(H)$ (by smoothness of $\phi$ ), and that for each $k \in K$, (48) allows us to drop all terms for interior faces that do not belong to some $\partial \tilde{I}_{k}^{i \pm}$, at the cost of terms of size $O(\rho) H^{d} \cdot O(H)=O(\rho) H^{d+1}$ per $k$, hence $O(\rho)$ overall. Here, it is important that $E_{F}\left(u^{h}\right)=O\left(h^{d}\right)$ (by (13)).

Moreover, by Lemma 6 the number of bad cubes is $o_{\rho, \epsilon}(1) H^{-(d+1)}$, and due to uniform boundedness of (13), (10), and (45),

$$
\sum_{C \rightarrow N \in \partial \tilde{I}_{k}}\left|E_{C \rightarrow N}\left(u^{h}\right)\right|=O\left(H^{d}\right) .
$$

Since the $\phi$ difference supplies an extra $O(H)$, the sum of terms in (67) where $k_{C}$ or $k_{N}$ is in $B$ is $o_{\rho, \epsilon}(1) H^{-(d+1)} \cdot O\left(H^{d}\right) \cdot O(H)=o_{\rho, \epsilon}(1)$. Hence the interior face 
part of (65) is

$$
\begin{aligned}
& =\sum_{i=0}^{d} \sum_{k \in G} \sum_{F \in \partial \tilde{I}_{k+e^{i-}}^{(i)}} E_{F}\left(u^{h}\right) \underbrace{\left(\phi\left(H\left(k+e^{(i)}\right)\right)-\phi(H k)\right)}+O(\rho)+o_{\rho, \epsilon}(1) \\
& =\sum_{i=0}^{d} \sum_{k \in G} \underbrace{\sum_{F \in \partial \tilde{I}_{k+e^{i-}}} E_{F}\left(u^{h}\right) H^{-d} \int_{I_{k}} \frac{\partial \phi}{\partial y_{i}}(y) d V(y)+O(\rho)+o_{\rho, \epsilon}(1)}
\end{aligned}
$$

(using smoothness and compact support of $\phi$ )

$$
=\sum_{i=0}^{d} \sum_{k \in G} \underbrace{\sum_{F \in \partial \tilde{I}_{k+e^{i}}^{(i)}} E_{F}\left(\hat{\bar{u}}_{k}\right)} H^{-d} \int_{I_{k}} \frac{\partial \phi}{\partial y_{i}}(y) d V(y)+O(\rho+\epsilon)+o_{\rho, \epsilon}(1)
$$

(using the definition (59) of $G$ in uniform continuity (12), combined with (45))

$$
=\sum_{i=0}^{d} \sum_{k \in G} \underbrace{\int_{\bigcup_{F \in \partial \tilde{I}^{i-}}^{i-e^{(i)}}} F} \underbrace{} \eta\left(\bar{u}_{k}, \cdot\right) \cdot n d S H^{-d} \int_{I_{k}} \frac{\partial \phi}{\partial y_{i}}(y) d V(y)+O(\rho+\epsilon)+o_{\rho, \epsilon}(1)
$$

(using consistency (11))

$$
=\sum_{i=0}^{d} \sum_{k \in G} \eta\left(\bar{u}_{k}, H k\right) \cdot \underbrace{\int_{\bigcup_{F \in \partial \tilde{I}_{k+e^{i}(i)}}} n d S} H^{-d} \int_{I_{k}} \frac{\partial \phi}{\partial y_{i}}(y) d V(y)+O(\rho+\epsilon)+o_{\rho, \epsilon}(1)
$$

(using smoothness in $y$ of $\eta$ (note (39), (17)) with (45) and (10))

$$
\begin{aligned}
& \stackrel{(50)}{=}-\sum_{i=0}^{d} \sum_{k \in G} \underbrace{\eta_{i}\left(\bar{u}_{k}, H k\right) \int_{I_{k}} \frac{\partial \phi}{\partial y_{i}} d V(y)}+O(\epsilon+\rho)+o_{\rho, \epsilon}(1) \\
& =-\sum_{i=0}^{d} \underbrace{\int_{I_{k}} \frac{\partial \phi}{\partial y_{i}}(y) \eta_{i}(u(y), y) d V(y)+O(\epsilon+\rho)+o_{\rho, \epsilon}(1)}_{\sum_{k \in G}}
\end{aligned}
$$

(using the definition (59) of $G$ and smoothness in $y$ and $u$ of $\eta$ )

$$
\stackrel{\text { (60) }}{=}-\sum_{i=0}^{d} \sum_{k \in K} \int_{I_{k}} \frac{\partial \phi}{\partial y_{i}}(y) \eta_{i}(u(y), y) d V(y)+O(\epsilon+\rho)+o_{\rho, \epsilon}(1)
$$

$$
=-\int_{\mathbb{R}_{+}^{d+1}} \sum_{i=0}^{d} \frac{\partial \phi}{\partial y_{i}}(y) \eta_{i}(u(y), y) d V(y)+O(\epsilon+\rho)+o_{\rho, \epsilon}(1) .
$$


It remains to treat the left-hand side of (65). As for the flux integrals, we may omit the terms for $k \in B$, at a cost of $o_{\rho, \epsilon}(1) H^{-(d+1)} \cdot O\left(H^{d+1}\right)=o_{\rho, \epsilon}(1)$ (from (60) resp. (19) ), hence:

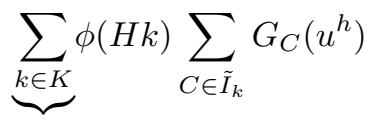

$$
\begin{aligned}
& =\sum_{k \in G} \phi(H k) \underbrace{\sum_{C \in \tilde{I}_{k}} G_{C}\left(u^{h}\right)}+o_{\rho, \epsilon}(1) \\
& \stackrel{[18)}{=} \sum_{k \in G} \phi(H k) \underbrace{\sum_{C \in \tilde{I}_{k}} G_{C}\left(\hat{\bar{u}}_{k}\right)}+O(\epsilon)+o_{\rho, \epsilon}(1)
\end{aligned}
$$

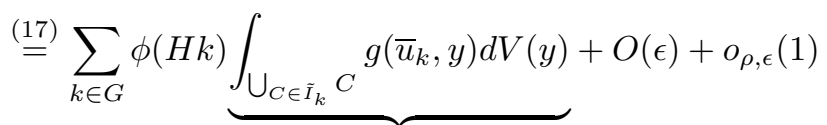

$$
\begin{aligned}
& =\sum_{k \in G} \phi(H k) \underbrace{V\left(\bigcup_{C \in \tilde{I}_{k}} C\right)} g\left(\bar{u}_{k}, H k\right)+O(\epsilon)+o_{\rho, \epsilon}(1) \\
& \stackrel{(38)}{=} \sum_{k \in G} \underbrace{\phi(H k) H^{d+1}} g\left(\bar{u}_{k}, H k\right)+O(\epsilon+\rho)+o_{\rho, \epsilon}(1) \\
& =\sum_{k \in G} \underbrace{\int_{I_{k}} \phi(y) d V(y) g\left(\bar{u}_{k}, H k\right)}+O(\epsilon+\rho)+o_{\rho, \epsilon}(1) \\
& =\underbrace{}_{\sum_{k \in G}} \int_{I_{k}} \phi(y) g(u(y), y) d V(y)+O(\epsilon+\rho)+o_{\rho, \epsilon}(1)
\end{aligned}
$$

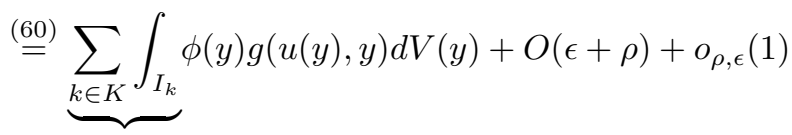

$$
\begin{aligned}
& =\int_{\mathbb{R}_{+}^{d+1}} \phi(y) g(u(y), y) d V(y)+O(\epsilon+\rho)+o_{\rho, \epsilon}(1) .
\end{aligned}
$$

3.5. Conclusion. Combining (66), (68), and (69), we get

$$
\begin{gathered}
\int_{\mathbb{R}_{+}^{d+1}} \phi(y) g(u(y), y) d V(y)+O(\epsilon+\rho)+o_{\rho, \epsilon}(1) \\
\geq-\int_{\mathbb{R}_{+}^{d+1}} \sum_{i=0}^{d} \frac{\partial \phi}{\partial y_{i}}(y) \eta_{i}(u(y)) d V(y)-\int \eta_{0}\left(u_{0}(x)\right) \phi(0, x) d S(x) .
\end{gathered}
$$


Now, we can first make the $O(\epsilon+\rho)$ term arbitrarily small by choosing appropriate $\epsilon$ and $\rho$; after that, the $o$ term can be made arbitrarily small as well by picking $H$. Therefore, $u$ satisfies (6); the proof is complete.

\section{A COUNTEREXAMPLE FOR NON-QUASI-UNIFORM GRIDS}

While most assumptions in this paper are rather weak, an important exception is quasi-uniformity (in the sense of (9)). Unfortunately, there is a strong counterexample to Theorem 1 for non-quasi-uniform grids (see Figure 4).

Example 1 (Staggered Lax-Friedrichs). Consider the trivial problem

$$
u_{t}=0, \quad u_{0}=\chi_{[0,1]} .
$$

We discretize it naïvely with the staggered Lax-Friedrichs scheme, for flux $f=0$, taking $\Delta_{t}=h^{3}$ where $h>0$ is the spatial cell size: let $\mathcal{C}^{h}$ contain the cells

$$
\begin{aligned}
E_{j}^{n} & :=[2 n h,(2 n+1) h] \times[j h,(j+1) h], \\
O_{j}^{n} & :=[(2 n+1) h,(2 n+2) h] \times\left[\left(j+\frac{1}{2}\right) h,\left(j+\frac{3}{2}\right) h\right] \quad\left(n \in \mathbb{N}_{0}, j \in \mathbb{Z}\right) .
\end{aligned}
$$

Define

$$
\begin{gathered}
F_{E_{j}^{n} \rightarrow O_{j}^{n}}\left(u^{h}\right)=F_{E_{j}^{n} \rightarrow O_{j-1}^{n}}=\frac{h}{2} u_{E_{j}^{n}}^{h}, \\
F_{O_{j}^{n} \rightarrow E_{j}^{n+1}}\left(u^{h}\right)=F_{O_{j}^{n} \rightarrow E_{j+1}^{n+1}}=\frac{h}{2} u_{O_{j}^{n}}^{h} \quad\left(n \in \mathbb{N}_{0}, j \in \mathbb{Z}\right), \\
F_{\partial \rightarrow E_{j}^{0}}\left(u^{h}\right)=\int_{2 j h}^{(2 j+1) h} u_{0}(x) d x \quad(j \in \mathbb{Z}), \\
F_{E_{j}^{n} \rightarrow E_{j+1}^{n}}\left(u^{h}\right)=0, \quad F_{O_{j}^{n} \rightarrow O_{j+1}^{n}}\left(u^{h}\right)=0 \quad\left(n \in \mathbb{N}_{0}, j \in \mathbb{Z}\right), \\
u_{E_{j}^{0}}:=h^{-1} \int_{j h}(j+1) h u_{0}(x) d S(x) .
\end{gathered}
$$

It is easy to check that the numerical fluxes are consistent and satisfy the initial condition; all other requirements are satisfied as well. However, the (uniquely

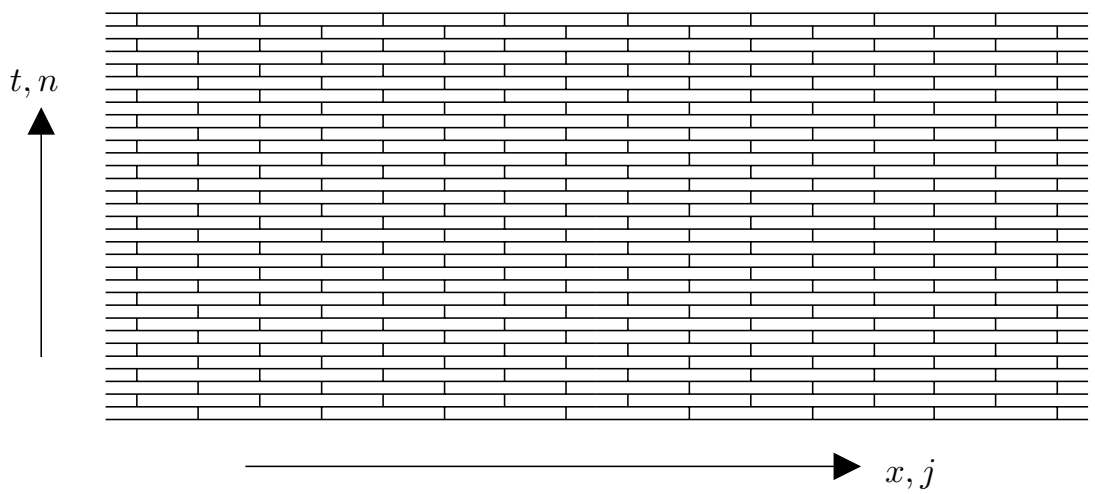

FiguRE 4. Staggered Lax-Friedrichs with $\Delta t=h^{3}, \Delta x=h$ : excessive refinement in the $t$ direction causes oversmoothing and convergence to a nonsolution. 
determined) solutions $\left(u^{h}\right)_{h>0}$ at a fixed time $t$ approximate $G\left(\frac{t}{4 h}, \cdot\right) * \chi_{[0,1]}$ (as $h \downarrow 0$ ), where $G$ is the heat kernel (this is easy to prove by considering two steps of the numerical scheme:

$$
u_{E_{j}^{n+1}}^{h}=\frac{u_{E_{j-1}^{n}}^{h}+2 u_{E_{j}^{n}}^{h}+u_{E_{j+1}^{n}}^{h}}{4}=u_{E_{j}^{n}}^{h}+\frac{\Delta_{t}}{4 h} \frac{u_{E_{j-1}^{n}}^{h}-2 u_{E_{j}^{n}}^{h}+u_{E_{j+1}^{n}}^{h}}{h^{2}} ;
$$

this is a well-known finite difference scheme for $u_{t}=\frac{1}{h} u_{x x}$, so standard theory applies). However, $\frac{t}{2 h} \rightarrow \infty$, so the $u^{h}$ converge in $L_{\text {loc }}^{1}$ to 0 , not to the actual solution $\chi_{[0,1]}$.

The example is so "economical" that it rules out any conceivable relaxation of the quasi-uniformity requirement: the cells are identical rectangles (in particular they are convex and have well-behaved surfaces) with sides parallel to the coordinate axes, numerical fluxes through a face depend only on one of the directly adjacent cells, and the numerical solutions $u^{h}$ are uniquely defined. The only "violation" is that cells become small in time direction faster than in space direction.

The example reflects a problem that does not appear in discretizations of semidiscrete schemes with artificial viscosity; while the viscosity coefficient of LaxFriedrichs type schemes is $\alpha h^{2} / \Delta_{t}$ (for some constant $\alpha$ ), it is typically $\alpha h$ for semidiscrete methods, so no harm can be done by choosing $\Delta_{t}$ too small.

Note that Noe95 presents a related counterexample, namely the Lax-Friedrichs scheme on a uniform Cartesian (nonstaggered) grid with $\Delta t / h \downarrow 0$ as $h \downarrow 0$. In contrast to staggered Lax-Friedrichs, this counterexample does not serve our purposes: for such a scheme, the flux between two intervals in a time step is not $O(\Delta t)$, as required by uniform boundedness (13), but $O(1)$.

The restriction to quasi-uniform grids is a serious one; results for non-quasiuniform grids are highly desirable because such grid sequences are produced by adaptive refinement and/or adaptive time integration. However, Theorem 1 probably remains true in a special case: tensor products of quasi-uniform grids, i.e.,

$$
\mathcal{C}^{h}=\bigotimes_{\alpha=1}^{s} \mathcal{C}_{\alpha}^{h}:=\left\{\prod_{\alpha=1}^{s} C_{\alpha}: C_{\alpha} \in \mathcal{C}^{h}\right\}
$$

where each $\mathcal{C}_{\alpha}^{h}$ is a grid of the type defined in Section 2.2 (for $\alpha=1$, the grid has to cover $\mathbb{R}_{+}^{d_{1}}$, for $\alpha>1$ the grid covers $\mathbb{R}^{d_{\alpha}}$ ). The case of semidiscrete schemes can be reduced to the tensor grid case. These questions will be explored in forthcoming work (see [Ella]).

For arbitrary non-quasi-uniform grids, on the other hand, it is necessary to impose stronger conditions on the numerical scheme. For example, one could study the error estimators that are used by adaptive schemes to determine where to refine the grid or decrease the time step, in order to derive additional smoothness or convergence information about $\left(u^{h}\right)_{h>0}$. If sufficiently weak assumptions can be made about a large class of error estimators, it might be possible to derive a Lax-Wendroff type result for non-quasi-uniform grids that is general enough to be interesting.

\section{NOVEl APPLiCATions}

5.1. Local time stepping. In order to resolve shocks or contact discontinuities well, it is necessary to refine the grid near them. The time step is limited by the CFL condition in small cells near these discontinuities and might be unnecessarily small 
for other parts of the domain. For this reason, it can be efficient to use different time steps in different regions; some schemes in this spirit have been proposed in OS83 or [Ell00, Chapter 4]. Theorem 1 is not limited to spatially unstructured grids; grids can be unstructured in space-time, as long as they are quasi-uniform in the sense of (9).

5.2. Moving vertices. Another use for the generalized Lax-Wendroff theorem is the large class of numerical methods with unsteady grids (see [Ell00, Section 2.1] for adaptation of classical approximate Riemann solvers to this case). These are important because some applications have moving domain boundaries, e.g., due to wing flutter or rotating turbine blades. Moreover, it is often natural or (for high Mach number supersonic flow) more efficient to use Lagrangian methods (grid vertices move along with the fluid). To accomodate these methods is straightforward: instead of a tensor product of time axis partition and fixed spatial grid, a grid with moving cells is used; faces are no longer either perpendicular or parallel to the time axes. (However, whether Theorem 1 is applicable depends on other details of the scheme as well.)

5.3. Conservative remapping. In numerical computations, it is sometimes necessary to change grids (remapping), for example because the old grid has developed singularities (especially common for Lagrangian schemes when there is strong vorticity in the flow field). See [Duk84, DK87, Gra99, Jon99, DB00 for remapping algorithms and applications. The remapping step should be conservative, for the same reasons that numerical schemes are conservative, and conservative quantities should not be "transported" during the remapping step more than necessary. A simple way to achieve this is to set

$$
u_{N}=V(N)^{-1} \sum_{O} V(O \cap N) u_{O},
$$

where $N$ is a cell in the new grid, $O$ runs over the cells in the old grid, and $u_{O}, u_{N}$ are densities of conserved quantities in each. (However, to achieve higher orders of accuracy, it might be necessary to compute polynomial or spline reconstructions $v(x)$ from the cell averages $u_{O}$ and to set

$$
u_{N}:=f_{N} v(x) d V(x) ;
$$

the previous scheme corresponds to the obvious piecewise constant reconstruction.)

It is not clear whether remapping can prevent an otherwise fine numerical scheme from converging to the entropy solution. However, Theorem 1 can be applied to answer this question for conservative remapping: the remapping step is interpreted as an extra hyperplane of faces (perpendicular to the time axis), with numerical fluxes defined depending on the remapping algorithm. The following requirements are weak enough to cover most existing methods:

(1) Consistency: whenever all $u_{O^{\prime}}$ are constant $=w$, the reconstruction $v$ should be constant $=w$ in each cell $O$.

(2) Continuity: the reconstruction map $u^{h} \mapsto v$ should be continuous on the "diagonal" of constant grid functions in the $L^{\infty} \rightarrow L^{\infty}$ topology. 
(3) Boundedness: the reconstruction map should be uniformly bounded; more precisely, for any $M$ there should be a constant $c$ so that

$$
\sup _{O}\left|u_{O}\right| \leq M \quad \Rightarrow \quad \int_{O}|v(x)| d V(x) \leq c V(O) .
$$

(4) Locality: the values of $v$ over a cell $O$ should depend only on cell averages $u_{O^{\prime}}$ for $d\left(O^{\prime}, O\right) \leq c h, c$ some constant independent of $h, O, O^{\prime}$.

(5) Conservation: the reconstruction $v$ should satisfy

$$
S(O) u_{O}^{h}=\int_{O} v(x) d V(x) .
$$

For every remapping step at some time $t$, the old and new cells generate a layer of faces. For every cell $N$ that meets a cell $O^{\prime}$ with $d\left(O^{\prime}, O\right) \leq C h$, add a face $O \rightarrow N$ and set

$$
F_{O \rightarrow N}=f_{N} v(x) d V(x) .
$$

By the assumptions, $F$ has bounded stencil, is consistent, uniformly continuous, and uniformly bounded; defining $F_{N \rightarrow O}:=-F_{O \rightarrow N}$ renders it conservative. Moreover, it is clear that if the old and new grids satisfy the requirements outlined in the Introduction and if the remapping steps are at least $\Omega(h)$ apart, then the resulting space-time grid is quasi-uniform.

This technique will be discussed in more detail in future work (see [Ellb]).

5.4. Self-similar flow. Self-similar solutions, i.e., those that satisfy $u(t, x)=$ $u(s t, s x)$ for all $s>0$, arise in many important circumstances, such as shocks, contacts or rarefaction waves Riemann problems, or in [Ell03. A self-similar solution to $u_{t}+\operatorname{div} f(u)=0$ satisfies the system

$$
\operatorname{div}_{\xi}(f(u)-\xi u)=-d u,
$$

where $\xi=\frac{x}{t}$ are called similarity coordinates. To find asymptotically stable selfsimilar solutions, one can solve

$$
u_{\tau}+\operatorname{div}_{\xi}(f(u)-\xi u)=-d u
$$

(where $\tau$ is a time-marching "pseudo-time" without physical significance). Adding the source term $-d u$ to numerical schemes in a conservative and consistent way (as defined in Section 2.4) is easy; achieving stability is not difficult either. Theorem 1 states that such a scheme delivers entropy solutions as long as it converges.

\section{REFERENCES}

[BL97] A. Bressan and P. LeFloch, Uniqueness of weak solutions to systems of conservation laws, Arch. Rat. Mech. Anal. 140 (1997), 301-317. MR1489317 (98m:35125)

[CCL94] B. Cockburn, F. Coquel, and P. LeFloch, An error estimate for finite volume methods for multidimensional conservation laws, Math. Comp. 63 (1994), 77-103. MR.1240657 (95d:65078)

[CL91] F. Coquel and P. LeFloch, Convergence of Finite Difference Schemes for Conservation Laws in Several Space Dimensions: The Corrected Antidiffusive Flux Approach, Math. Comp. 57 (1991), no. 195, 169-210. MR.1079010 (91m:65229)

[CL93] Convergence of Finite Difference Schemes for Conservation Laws in Several Space Dimensions: A General Theory, SIAM J. Numer. Anal. 30 (1993), no. 3, 675-700. MR.1220646 (94e:65092)

[CM80] M.G. Crandall and A. Majda, Monotone difference approximations for scalar conservation laws, Math. Comp. 34 (1980), no. 149, 1-21. MR0551288 (81b:65079) 
[CT80] M.G. Crandall and L. Tartar, Some relations between nonexpansive and order preserving mappings, Proc. AMS 78 (1980), no. 3, 385-390. MR0553381 (81a:47054)

[DB00] J. K. Dukowicz and J. R. Baumgardner, Incremental remapping as a transport/advection algorithm, J. Comput. Phys. 160 (2000), 318-335.

[DK87] J. K. Dukowicz and J. W. Kodis, Accurate conservative remapping (rezoning) for arbitrary Lagrangian-Eulerian computations, J. Comput. Phys. 8 (1987), no. 3, 305-321. MR0883773 (88b:76008)

[Duk84] J. K. Dukowicz, Conservative rezoning (remapping) for general quadrilateral meshes, J. Comput. Phys. 54 (1984), 411-424.

[Ella] V. Elling, A Lax-Wendroff type theorem for semidiscrete schemes on unstructured quasi-uniform grids, in preparation.

[Ellb] Methods and theory for conservative remapping, in preparation.

[Ell00] - Numerical simulation of gas flow in moving domains, Diploma Thesis, RWTH Aachen (Germany), 2000.

[Ell03] A possible counterexample to entropy solutions and to Godunov scheme convergence, 75 (2006), 1721-1733.

[GR96] E. Godlewski and P.-A. Raviart, Numerical approximation of hyperbolic systems of conservation laws, Springer, 1996. MR.1410987 (98d:65109)

[Gra99] J. Grandy, Conservative remapping and region overlays by intersecting arbitrary polyhedra, J. Comput. Phys. 148 (1999), 433-466. MR.1669715 (99i:76114)

[HHL76] A. Harten, J.M. Hyman, and P.D. Lax, On finite-difference approximation and entropy conditions for shocks, Comm. Pure Appl. Math. 29 (1976), 297-321. MR0413526 $(54: 1640)$

[Jon99] P.W. Jones, First- and second-order conservative remapping schemes for grids in spherical coordinates, Monthly Weather Review (1999), no. 9, 2204-2210.

[Kru70] S.N. Kružkov, First order quasi-linear equations in several independent variables, Mat. Sb. 81 (1970), no. 2, 285-355, transl. in Math. USSR Sb. 10 (1970) no. 2, 217-243. MR0267257 (42:2159)

[KRW96] D. Kröner, M. Rokyta, and M. Wierse, A Lax-Wendroff type theorem for upwind finite volume schemes in 2-D, East-West J. Numer. Math. 4 (1996), 279-292. MR1430241 (97m:65158)

[Kuz75] N.N. Kuznetsov, On stable methods for solving a first-order quasi-linear equation in the class of discontinuous functions, Dokl. Akad. Nauk. SSSR 225 (1975), no. 5, 25-28, transl. in USSR Comp. Math. and Math. Phys. 16 (1976) no. 6, 105-119. MR0445103 $(56: 3448)$

[LeV92] R.J. LeVeque, Numerical methods for conservation laws, 2nd ed., Birkhäuser, 1992. MR.1153252 (92m:65106)

[LW60] P. Lax and B. Wendroff, Systems of conservation laws, Comm. Pure Appl. Math. 13 (1960), 217-237. MR0120774(22:11523)

[Noe95] S. Noelle, Convergence of higher order finite volume schemes on irregular grids, Adv. Comp. Math. 3 (1995), 197-218. MR1325031(96a:65137)

[OS83] S. Osher and R. Sanders, Numerical approximations to nonlinear conservation laws with locally varying time and space grids, Math. Comp. 41 (1983), no. 164, 321-336. MR 0717689(85i:65121)

[San83] R. Sanders, On convergence of monotone finite difference schemes with variable spatial differencing, Math. Comp. 40 (1983), no. 161, 91-106. MR0679435 (84a:65075)

Division of Applied Mathematics, Brown University, 182 George Street, Providence, RHODE ISLAND 02906

E-mail address: velling@stanfordalumni.org

URL: http://www.dam.brown.edu/people/volker 